\title{
Human computation for constraint-based recommenders
}

\author{
Thomas Ulz ${ }^{1}$ Michael Schwarz ${ }^{1}$. \\ Alexander Felfernig ${ }^{1} \cdot$ Sarah Haas $^{1}$ - Amal Shehadeh ${ }^{1}$. \\ Stefan Reiterer ${ }^{1} \cdot$ Martin Stettinger ${ }^{1}$
}

Received: 8 December 2015 / Revised: 11 September 2016 / Accepted: 15 September 2016 /

Published online: 28 September 2016

(C) The Author(s) 2016. This article is published with open access at Springerlink.com

\begin{abstract}
PeopleViews is a Human Computation based environment for the construction of constraint-based recommenders. Constraint-based recommender systems support the handling of complex items where constraints (e.g., between user requirements and item properties) can be taken into account. When applying such systems, users are articulating their requirements and the recommender identifies solutions on the basis of the constraints in a recommendation knowledge base. In this paper, we provide an overview of the PEOPLEVIEWS environment and show how recommendation knowledge can be collected from users of the environment on the basis of micro-tasks. We also show how PEOPLEVIEWS exploits this knowledge for automatically generating recommendation knowledge bases. In this context, we compare the prediction quality of the recommendation approaches integrated in PEOPLEVIEWS using a DSLR camera dataset.
\end{abstract}

Alexander Felfernig

alexander.felfernig@ist.tugraz.at

Thomas Ulz

thomas.ulz@student.tugraz.at

Michael Schwarz

michael.schwarz@student.tugraz.at

Sarah Haas

sarah.haas@student.tugraz.at

Amal Shehadeh

amal.shehadeh@ist.tugraz.at

Stefan Reiterer

stefan.reiterer@ist.tugraz.at

Martin Stettinger

martin.stettinger@ist.tugraz.at

1 Applied Software Engineering Group, Institute for Software Technology, TU Graz, Graz, Austria 
Keywords Constraint-based systems $\cdot$ Recommender systems $\cdot$ Constraint-based recommendation $\cdot$ Human computation $\cdot$ Knowledge acquisition

\section{Introduction}

In contrast to collaborative filtering (Goldberg et al. 1992; Konstan et al. 1997), contentbased filtering (Pazzani and Billsus 1997; Roy and Mooney 2004), and case-based recommendation (Burke and Hammond 1997; McCarthy et al. 2005; Musto et al. 2014), constraint-based recommender systems (as a specific type of knowledge-based recommender system) (Burke 2000; Felfernig and Burke 2008; Jannach et al. 2010) rely on a predefined set of constraints that perform the selection of candidate items (Burke and Ramezani 2010). Constraints make it possible to explicitly define specific domain properties and restrictions which is not possible in recommendation approaches such as collaborative filtering, content-based filtering, and case-based recommendation (Jannach et al. 2010; Ricci et al. 2011). Especially for complex items, content-based and collaborative filtering approaches can not be directly applied, since these approaches do not allow the inclusion of rules and/or constraints. However, such an inclusion of constraints comes along with a major challenge which is the knowledge acquisition bottleneck: domain experts and knowledge engineers have to extensively communicate to correctly encode the recommendation knowledge. The PEOPLEVIEWS approach ${ }^{1}$ to generate constraints out of user feedback aims to reduce overheads related to the communication between domain experts and engineers.

Constraint-based recommender systems are applied in more complex item domains where a user specifies a set of criteria (Felfernig and Burke 2008; Felfernig et al. 2009) and the system proposes a corresponding set of recommendations. Items in these domains are purchased less frequently which makes traditional recommendation approaches such as collaborative filtering less applicable (Felfernig and Burke 2008). Constraint-based recommenders are typically conversational (Salem et al. 2014), i.e., the user provides answers to a set of questions and the system tries to retrieve relevant items. Constraints define restrictions regarding the possible combinations of customer requirements (e.g., a pocket camera does not support exchangeable lenses) and the relationships between customer requirements and item properties (e.g., the price of the recommended item must not exceed the maximum price specified by the customer). Constraints are used to pre-filter candidate items which are then ranked on the basis of a utility scheme provided, for example, by multi-attribute utility theory (Felfernig et al. 2006; Winterfeldt and Edwards 1986).

Constraint-based recommender systems are applied in various domains. Peischl et al. (2010) present an application of constraint-based recommendation technologies for supporting the selection of software testing methods. Felfernig et al. (2006) report applications of these technologies in the domain of financial services and electronic equipment. Leitner et al. (2012) show the application of constraint-based recommendation for recommending smart home solutions to users. WEEVIS ${ }^{2}$ (Reiterer 2015) is a constraint-based environment embedded in MediaWiki ${ }^{3}$ which allows the definition of recommender applications with a wiki-style syntax and the integration of these recommenders into standard Wiki

\footnotetext{
${ }^{1}$ The work presented in this article has been developed within the scope of the PEOPLEVIEWS project which is funded by the Austrian Research Promotion Agency (843492).

${ }^{2}$ www.weevis.org.

${ }^{3}$ www.mediawiki.org.
} 
pages. Jannach and Bundgaard-Joergensen (2007) introduce an application that focuses on the recommendation of business plans for company founders. Finally, Torrens et al. (2003) introduce an environment for the planning and recommendation of travel itineraries which is based on soft constraint technologies used for determining preferred solutions.

Development processes related to constraint-based recommender systems often come along with the so-called knowledge acquisition bottleneck where knowledge engineers are overwhelmed by the increasing size and complexity of knowledge bases. In this paper we show how Human Computation concepts (von Ahn 2005) can be employed to extract recommendation knowledge directly from users and domain experts and thus to lift the burden of effortful engineering from the knowledge engineer's shoulders. The major idea of Human Computation (Shah and Zhou 2015; von Ahn 2005) is to let humans perform simple and shortterm tasks they are better in compared to computers - in the context of knowledge engineering scenarios, the overall idea is to let domain experts perform simple and shortterm knowledge engineering tasks they are much better in, compared to knowledge engineers.

The idea of PEOPLEVIEWS is to engage domain experts (e.g., sales representatives) more deeply into knowledge engineering processes by making the corresponding engineering tasks more accessible to them. This is achieved via so-called micro-tasks that are basic tasks which can be completed within a short period of time. User feedback on micro-tasks is exploited for deriving recommendation rules (filter constraints) that help to select items. The potential advantages of applying PEOPLEVIEWS technologies are less efforts related to recommendation knowledge base development and maintenance, fewer erroneous constraints (compared to knowledge engineers, domain experts know more about the item domain), and a significantly higher degree of scalability, i.e., more recommenders can be maintained in parallel (which could not be achieved by a small number of knowledge engineers).

Applying Human Computation (von Ahn 2005) in the context of recommender systems development is not new. Hacker and Matchin (2009) introduce the MATCHIN environment where the elicitation of preferences from users is based on the idea of asking questions on which decisions other users would take when being confronted with a set of items. Walsh and Golbeck (2009) introduce a game environment (CURATOR) which can be used to configure item collections. The overall goal is, for example, to enforce users to develop a common understanding of a reasonable item collection. Colson (2013) introduces a personalized styling service for apparel items. In this context, a hybrid approach (combination of human computation and machine learning) is applied to determine recommendations. Finally, Larson et al. (2013) introduce the idea of not only recommending items to users but also users to items in situations where additional evaluations are needed in order to deal with sparse data and thus to improve recommendation quality. In the line of the term useritem reciprocity introduced by Larson et al. (2013) and Said et al. (2014), PEOPLEVIEWS does not only support the recommendation of items but also the recommendation of users who should provide item evaluations - this is also denoted as task routing. Solutions for task routing in micro-task environments have been presented, for example, in Jung (2014) who introduces a matrix factorization (Koren et al. 2009) based approach to learn reasonable assignments of micro-tasks to users. A corresponding key insight is that task similarity is a core factor in identifying appropriate task routings.

In PEOPLEVIEWS, task similarity is taken into account by determining the contentdependent similarity between already completed tasks of a user (the corresponding information is stored in the user profile) and a new task. The assignment of tasks to users is also addressed in the context of active learning (Elahi et al. 2016) which represents the task of selecting items that should be rated by a user with the goal to improve the overall recommendation accuracy. Further related work on task routing can be found in 
Cosley et al. (2007) and Iren and Bilgen (2014). For a detailed discussion of the micro task scheduling approaches included in PEOPLEVIEWS we refer to Felfernig et al. (2015). Nasery et al. (2014) compare true user preferences on features with those predicted by the recommender system. The outcome of their analysis is that there exists a significant mismatch between these two types of preferences. These results justify investments related to the crowd-sourced collection of user item evaluations. A major difference between PEOPLEVIEWS and related work is the focus on the derivation of constraints which enables conversational recommendation and repair processes (Felfernig et al. 2006). Although also operating on user queries, the major difference between PEOPLEVIEWs compared to tagging-based recommendation approaches (Zanardi and Cara 2008) is the focus on similarity metrics when determining recommendations; again, no constraint-based mechanisms such as inconsistency checking and consistency restoration can be applied in tagging-based approaches.

The contributions of this paper are the following. First, we introduce a new approach to the development of knowledge bases for constraint-based recommendation scenarios. This approach helps to assure scalability since it makes it possible to also engage domain experts without a computer science background into knowledge engineering tasks. Second, we introduce new user interfaces that support the acquisition of recommendation knowledge on the basis of micro-tasks. Third, we introduce a new type of constraint representation that allows to automatically derive constraints from user item evaluations. Fourth, we report the results of an empirical study that compares the prediction quality of the included recommendation approach with some baseline versions (random, most popular, and user similarity based recommendation). Finally, we discuss a couple of relevant issues for future work.

The remainder of this paper is organized as follows. In Section 2 we show how recommender knowledge bases can be defined in the PEOPLEVIEWS environment. Thereafter we provide an overview of the current version of the PEOPLEVIEWS user interface (Section 3). In Section 4 we compare recommendation approaches currently implemented in PEOPLEVIEWS with regard to their predictive quality. In Section 5 we provide an overview of ongoing and future work. With Section 6 we conclude the paper.

\section{Recommendation approach}

When applying a PEOPLEVIEWs recommender, users are specifying their preferences (requirements) in terms of user attribute values that are in the following translated into corresponding item recommendations. An example set of user attributes $(u \in U)$ is depicted in Table 1: application (single-valued) represents the preferred application of a digital camera, usertype specifies the type of user the camera is suited for, and usability specifies the minimum degree of usability a user is willing to accept. Each user attribute has an associated choice type (single or multiple choice), a question that is posed to the user, and an attribute domain definition (enum, integer, boolean, and text). An example set of user requirements $\left(r e q_{i} \in R E Q\right)$, i.e., instantiations of attributes specified by a user, is depicted in Table 2.

In addition to user attributes, product attributes describe technical properties of an item. This distinction between the user view and the technical item view is frequently applied in knowledge-based recommendation and configuration scenarios - see, for example, Felfernig et al. (2006) and Mittal and Frayman (1989). Table 3 depicts a set of example product attributes of a digital camera which include sensorsize, maxshutterspeed, maxISO, maxresolution, and price (a corresponding item set is depicted in Table 4). In addition to the choice type and the question posed to a recommender user (technical properties of items can also 
Table 1 Example user attributes $(u \in U)$ of a PEOPLEVIEWS digital camera recommender

\begin{tabular}{|c|c|c|c|c|}
\hline attribute & explanation & choice type & question to user & domain \\
\hline application & $\begin{array}{l}\text { application } \\
\text { domains }\end{array}$ & single & $\begin{array}{l}\text { Preferred } \\
\text { Application? }\end{array}$ & $\begin{array}{l}\text { \{sport, architecture, macro, } \\
\text { landscape, portrait\} }\end{array}$ \\
\hline usertype & $\begin{array}{l}\text { photography } \\
\text { experiences }\end{array}$ & multiple & Suited for whom? & \{beginner, amateur, expert\} \\
\hline usability & $\begin{array}{l}\text { usability of } \\
\text { digital camera }\end{array}$ & single & $\begin{array}{l}\text { Minimum accepted } \\
\text { usability? }\end{array}$ & \{average, high, very high\} \\
\hline
\end{tabular}

be specified via a search interface), each product attribute is associated with a corresponding attribute level similarity metric (McSherry 2003). The metrics can be exploited to determine items that are similar compared to the criteria (product attribute properties) specified by the user. In this context, the equal is better (EIB) metric denotes the fact that two attribute values are only similar if they have the same value; nearer is better (NIB) denotes the fact that the lower the distance between two attribute values, the better. Furthermore, more is better (MIB) denotes the fact that the higher the value of an item the better, and less is better (LIB) denotes the fact that the lower the value the better. These metrics will be explained in more detail in the context of the PEOPLEVIEWS recommendation strategies (see Formula (5)).

User attributes as well as product attributes can only be defined by the creator of a recommender application - related requests for additional attributes or changes in the domain of an attribute can be posed in the PEOPLEVIEWs forum. Users of the PEOPLEVIEWS community are free to integrate additional items - in this context, each item (product) attribute value has to be specified. PEOPLEVIEWs users are also in charge of specifying the relationship between user attributes and items. This is achieved through the completion of micro-tasks, for example, a user has to evaluate to which extent a digital camera supports users who are beginners in digital photography (i.e., usertype = beginner). The "wisdom of the crowd" is then exploited for deriving a corresponding recommendation rule (filter constraint), for example, if the majority of users provide a low evaluation for usertype = beginner for the digital camera Canon EOS 5D Mark III, the corresponding recommender will not regard the camera as best candidate for beginners. Users of a PEOPLEVIEWS recommender can add recommendation knowledge via providing feedback on assigned micro-tasks or by proactively evaluating items.

PEOPleViews supports two interaction modes. First, PEOPLEVIEWs recommenders support users in finding a product (item) that fits their wishes and needs (recommendation mode) - in this context, user and product attributes are used to specify user (customer) requirements $r e q_{i} \in R E Q$. Second, recommenders can be developed in the modeling mode. In this mode, user attributes are central elements used in a micro-task: given a certain item, users estimate which values of a user attribute fit the item to which extent. There are different types of micro-tasks used to figure out which user attribute settings are compatible with

Table 2 Example user requirements $r e q_{i} \in R E Q$

\begin{tabular}{lll}
\hline id & user attribute & value \\
\hline$r e q_{1}$ & application & sport \\
$r e q_{2}$ & usertype & expert \\
$r e q_{3}$ & usability & very high \\
\hline
\end{tabular}


Table 3 Example product attributes $(p \in P$ ) of a PEOPLEVIEWs digital camera recommender

\begin{tabular}{|c|c|c|c|c|c|}
\hline attribute & $\begin{array}{l}\text { choice } \\
\text { type }\end{array}$ & $\begin{array}{l}\text { question to } \\
\text { recommender } \\
\text { user }\end{array}$ & domain & $\begin{array}{l}\text { similarity } \\
\text { metric }\end{array}$ & $\begin{array}{l}\text { show to } \\
\text { user? }\end{array}$ \\
\hline sensorsize & single & $\begin{array}{l}\text { Preferred sensor } \\
\text { size? }\end{array}$ & $\begin{array}{l}\text { fullframe, APS-C, } \\
\text { MFT, 1", } 2 / 3 ”\}\end{array}$ & EIB & yes \\
\hline maxshutterspeed & single & $\begin{array}{l}\text { Required max. } \\
\text { shutter speed? }\end{array}$ & $\begin{array}{l}\{1 / 4.000,1 / 6.000, \\
1 / 8.000,1 / 16.000\}\end{array}$ & MIB & no \\
\hline $\max I S O$ & single & $\begin{array}{l}\text { Required max. } \\
\text { ISO sensitivity? }\end{array}$ & $\begin{array}{l}\{6.400,12.800, \\
25.600\}\end{array}$ & MIB & no \\
\hline maxresolution & single & Max. resolution? & $\begin{array}{l}\text { \{8 Mpix, } 12 \text { Mpix, } \\
\text { 16Mpix, 18Mpix, } \\
21 \text { Mpix, 23Mpix }\}\end{array}$ & MIB & yes \\
\hline price & single & Max. price? & integer & LIB & no \\
\hline
\end{tabular}

which items - details on the different types of PEOPLEVIEWS micro-tasks will be given in Section 3. An example of the evaluation of items with regard to the given set of user attribute values is depicted in Table 5 - in PEOPLEVIEWS, only logged-in users are allowed to enter evaluation data (one evaluation per item and user attribute). For example, Giselle rates the macro capabilities of item $\Phi_{3}$ as very high (user-specific evaluation: 1.0). Userspecific evaluations indicate on a scale [0..1] the degree to which an item supports a given attribute value from a specific user's point of view.

Each entry in Table 5 represents one user-specific filter constraint which specifies selection criteria for one item from the viewpoint of an individual user. For example, user Giselle evaluates the Canon EOS 5D Mark III (item $\Phi_{3}$ ) as an excellent candidate (userspecific evaluation: 1.0) for macro photography. Furthermore, Giselle thinks that the camera should only be recommended to experts (user-specific evaluation: 1.0), the usability of the camera is regarded as high (user-specific evaluation: 1.0). Since user-specific filter constraints only reflect the views of individual users, these constraints have to be aggregated. Recommendation-relevant filter constraints are aggregated user-specific filter constraints examples of this aggregation step are depicted in Table 6 . For $\Phi_{1}$, all related user-specific filter constraints are integrated into one recommendation-relevant filter constraint.

The user-specific evaluations (see Table 5) for an item $\Phi$, user attribute $u$, and user attribute value $v$ are aggregated into one recommendation-relevant evaluation (see Formulae (1) and (2)). This eval value is calculated from the average of all existing user-specific evaluations of attribute value $v$ of attribute $u$ of item $\Phi$ times the corresponding support. The support parameter is used to relativize a user-specific evaluation in terms of how often one attribute value for a specific item $\Phi$ has been selected by users compared to other values

Table 4 Example set of digital cameras defined using the product attributes of Table 3

\begin{tabular}{lllllll}
\hline item id & item & sensorsize & maxshutterspeed & maxISO & maxresolution & price \\
\hline$\Phi_{1}$ & Canon EOS 7D & APS-C & $1 / 8000$ & 12.800 & $18 \mathrm{Mpix}$ & 800 \\
$\Phi_{2}$ & Canon EOS 550D & APS-C & $1 / 4000$ & 6.400 & $18 \mathrm{Mpix}$ & 500 \\
$\Phi_{3}$ & Canon EOS 5D Mark III & fullframe & $1 / 8.000$ & 25.600 & $23 \mathrm{Mpix}$ & 2.000 \\
\hline
\end{tabular}


Table 5 User-specific evaluations (numbers in brackets) of cameras in PEOPLEVIEWS. Each row corresponds to a user-specific filter constraint

\begin{tabular}{|c|c|c|c|c|c|}
\hline id & user & item id & application & usertype & usability \\
\hline 1 & Jenny & $\Phi_{1}$ & sport (1.00) & amateur (0.60), expert (1.00) & high (0.95) \\
\hline 2 & Giselle & $\Phi_{1}$ & sport $(0.95)$ & amateur (0.50), expert (1.00) & high (1.0) \\
\hline 3 & Sarah & $\Phi_{1}$ & sport $(0.95)$ & amateur (0.75), expert (1.00) & very high (1.0) \\
\hline 4 & David & $\Phi_{1}$ & portrait $(0.80)$ & beginner $(0.00)$, amateur $(0.10)$, expert $(1.00)$ & high $(0.95)$ \\
\hline 5 & Mike & $\Phi_{1}$ & sport $(1.00)$ & beginner $(0.40)$, amateur $(0.80)$, expert $(1.00)$ & very high $(0.95)$ \\
\hline 6 & Jenny & $\Phi_{2}$ & landscape $(0.75)$ & beginner $(1.00)$, amateur $(0.80)$, expert $(0.00)$ & high $(0.95)$ \\
\hline 7 & Giselle & $\Phi_{2}$ & sport $(0.60)$ & beginner $(0.90)$, amateur $(0.00)$ & high $(0.90)$ \\
\hline 8 & Sarah & $\Phi_{2}$ & landscape $(0.80)$ & beginner $(1.00)$ & average (1.00) \\
\hline 9 & David & $\Phi_{2}$ & portrait $(0.70)$ & beginner $(0.90)$, amateur $(0.90)$ & high $(1.00)$ \\
\hline 10 & Mike & $\Phi_{2}$ & portrait $(0.75)$ & beginner $(1.00)$ & very high $(1.00)$ \\
\hline 11 & Jenny & $\Phi_{3}$ & sport $(0.80)$ & beginner $(0.10)$, amateur $(0.60)$, expert $(1.00)$ & very high $(1.00)$ \\
\hline 12 & Giselle & $\Phi_{3}$ & macro $(1.00)$ & expert $(1.00)$ & high (1.00) \\
\hline 13 & Sarah & $\Phi_{3}$ & macro $(0.90)$ & expert $(1.00)$ & very high $(0.90)$ \\
\hline 14 & David & $\Phi_{3}$ & macro $(0.95)$ & beginner $(0.30)$, amateur $(0.40)$, expert $(0.90)$ & very high $(0.90)$ \\
\hline 15 & Mike & $\Phi_{3}$ & portrait $(0.95)$ & amateur $(0.00)$, expert $(0.95)$ & high $(0.95)$ \\
\hline
\end{tabular}

of the same attribute, i.e., support in our context is an indication of how frequently a user attribute value evaluation for an item appears in the global evaluation set. For example, the support for the attribute value sport for item $\Phi_{3}$ is $\frac{1}{5}$. Finally, count $(\Phi, u, v)$ denotes the number of evaluations of attribute value $v$ for item $\Phi$ and $\operatorname{count}(\Phi, u)$ is the number of evaluations of attribute $u$ for item $\Phi$.

$$
\begin{gathered}
\operatorname{eval}(\Phi, u, v)=\frac{\operatorname{sum}_{v}(\Phi, u, v)}{\operatorname{count}(\Phi, u, v)} \times \operatorname{support}(\Phi, u, v) \\
\operatorname{support}(\Phi, u, v)=\frac{\operatorname{count}(\Phi, u, v)}{\operatorname{count}(\Phi, u)}
\end{gathered}
$$

Table 6 Example of recommendation-relevant filter constraints derived from user-specific filter constraints (see Table 5). User-specific evaluations (Table 5) are integrated into recommendation-relevant evaluations

\begin{tabular}{|c|c|c|c|c|}
\hline constraint & item name (id) & application & usertype & usability \\
\hline$f_{1}$ & Canon EOS 7D $\left(\Phi_{1}\right)$ & $\begin{array}{l}\text { sport }(0.78), \\
\text { portrait }(0.16)\end{array}$ & $\begin{array}{l}\text { beginner }(0.08) \text {, } \\
\text { amateur }(0.55) \text {, } \\
\text { expert }(1.0)\end{array}$ & $\begin{array}{l}\text { high }(0.58), \\
\text { very high }(0.39)\end{array}$ \\
\hline$f_{2}$ & Canon EOS 550D $\left(\Phi_{2}\right)$ & $\begin{array}{l}\text { landscape }(0.31) \text {, } \\
\text { sport }(0.12) \text {, } \\
\text { portrait }(0.29)\end{array}$ & $\begin{array}{l}\text { beginner }(0.96) \text {, } \\
\text { amateur }(0.34)\end{array}$ & $\begin{array}{l}\text { average }(0.2) \text {, } \\
\text { high }(0.57), \\
\text { very high }(0.2)\end{array}$ \\
\hline$f_{3}$ & Canon EOS 5D Mark III $\left(\Phi_{3}\right)$ & $\begin{array}{l}\text { sport }(0.16), \\
\text { macro }(0.57) \text {, } \\
\text { portrait }(0.19)\end{array}$ & $\begin{array}{l}\text { beginner }(0.08) \\
\text { amateur }(0.2) \text {, } \\
\text { expert }(0.97)\end{array}$ & $\begin{array}{l}\text { high }(0.39), \\
\text { very high }(0.56)\end{array}$ \\
\hline
\end{tabular}
(see eval in Formula (1)) - if eval $=0$, the corresponding attribute value is not part of the filter constraint) 
The recommendation-relevant filter constraints of Table 6 have to be interpreted, for example, as follows: item $\Phi_{1}$ (Canon EOS 7D) is included (recommended) if the specified requirements regarding the user attributes application, usertype, and usability are consistent with the condition in the corresponding recommendation-relevant filter constraint: application $\in\{$ sport, portrait $\} \wedge$ usertype $\in\{$ beginner, amateur, expert $\} \wedge$ usability $\in\{$ high, very high $\} \rightarrow$ include $\left(\Phi_{1}\right)$. This aggregation step has to be performed for each individual item - in our working example, the result of this aggregation step are three different recommendation-relevant filter constraints (each item has exactly one) which are then executed on the item table.

The logical representation of a recommendation-relevant filter constraint $f$ for an item $\Phi$ is depicted in Formula (3). In this formula, values $(\Phi, u)$ is the set of supported domain values (with eval $>0$ ) of user attribute $u \in U$, furthermore, noval denotes the fact that no value has been selected for the corresponding user attribute.

$$
f(\Phi): \bigwedge_{u \in U} u \in \operatorname{values}(\Phi, u) \cup\{\text { noval }\} \rightarrow \operatorname{include}(\Phi)
$$

Depending on the requirements articulated by the user $\left(r e q_{i} \in R E Q\right)$, PEOPLEVIEWS identifies and ranks items as follows. First, all recommendation-relevant filter constraints $f$ are used to preselect items (identification of a candidate set). On the basis of the user requirements defined in Table 2, the items $\left\{\Phi_{1}, \Phi_{3}\right\}$ can be identified as candidate items. The following utility function (Formula (4)) can now be used to rank the items in the candidate set. This way, the utility of each item is determined on the basis of the evaluations of user attribute values (see Formula (1)). The function $w(a)$ denotes an attribute weight which has been specified by a user or has been learned on the basis of already existing user interaction logs with the recommender.

$$
\text { utility }(\Phi, R E Q)=\Sigma_{a=v \in R E Q} \operatorname{eval}(\Phi, a, v) \times w(a)
$$

The item ranking in our working example determined on the basis of Formula (4) is the following. The utility of item $\Phi_{1}$ is $0.78+1.0+0.39=2.17$ whereas the utility of item $\Phi_{3}$ is $0.16+0.97+0.56=1.69$. Consequently, item $\Phi_{1}$ is ranked before item $\Phi_{3}$.

Up to now we did not take into account user requirements related to item properties, i.e., we discussed an example scenario that only takes into account the user requirements depicted in Table 2. If user requirements $\left(r e q_{i} \in R E Q\right)$ should also include requirements regarding item properties, we have to define the calculation of evaluations for items $\Phi$, product attributes $p$, and product attribute values $v$ (see Formula (5)). In PEOPLEVIEWS, such evaluations are determined, for example, on the basis of the attribute level similarity metrics Equal Is Better (EIB), Nearer Is Better (NIB), More Is Better (MIB), and Less Is Bet$\operatorname{ter}(L I B)$. A related overview of similarity metrics can be found, for example in McSherry (2003).

$$
\operatorname{eval}(\Phi, p, v)=\left\{\begin{array}{r}
1 \text { if } v=\operatorname{val}(\Phi, p), 0 \text { otherwise } \mathrm{EIB} \\
1-\frac{|v-\operatorname{val}(\Phi, p)|}{\max (\Phi, p)-\min (\Phi, p)} \mathrm{NIB} \\
\frac{\operatorname{val}(\Phi, p)-\min (\Phi, p)}{\max (\Phi, p)-\min (\Phi, p)} \mathrm{MIB} \\
\frac{\max (\Phi, p)-\operatorname{val}(p)}{\max (\Phi, p)-\min (\Phi, p)} \mathrm{LIB}
\end{array}\right.
$$

Improving the Basic Recommendation Approach using Beta Distributions. The afore discussed recommendation approach calculates the average evaluation values but does not take into account the aspect of representativity. For example, if camera $A$ has been evaluated by only one user and received an evaluation of $90 \%$ for the usage in landscape photography 
and camera $B$ already received 100 evaluations but has an average evaluation of $85 \%$ then an interpretation could be that the first camera is better in the dimension landscape photography. In order to take into account such situations, we employ beta distributions that can be used to describe the behavior of random variables. For the sample data of each attribute value and the corresponding item, we have to derive a corresponding beta distribution. The mode of each such distribution is the most likely value of the distribution and in our case corresponds to the average of the evaluations used to derive the distribution. Applying the beta distribution function to the determined mode value results in the corresponding peak value, i.e., the maximum value of the distribution.

The higher the peak value of a beta distribution related to a set of item $x$ attribute value evaluations, the higher the corresponding confidence. In this context, we interpret confidence as an indication of how often an attribute value is referred to by a user-specific evaluation. Formula (6) shows the calculation of recommendation-relevant evaluations $\left(\mathrm{eval}_{\beta}\right)$ when peak values of the underlying beta distributions are taken into account. This approach to determine item attribute evaluations will be compared with the aforementioned standard approach in Section 4. The underlying expectation is that taking into account the aspect of confidence leads to a better prediction quality of the recommendation approach.

$$
\begin{gathered}
\operatorname{eval}_{\beta}(\Phi, u, v)=\operatorname{eval}(\Phi, u, v) \times \operatorname{confidence}(\Phi, u, v) \\
\operatorname{confidence}(\Phi, u, v)=\frac{\operatorname{peak}(\Phi, u, v)}{\max (\operatorname{peaks}(\Phi, u, v))}
\end{gathered}
$$

\section{PeopleViews user interface}

In the PEOPLEVIEWS recommendation mode, users can specify their requirements (see Fig. 1) and PEOPLEVIEWS recommenders determine recommendations on the basis of recommendation-relevant filter constraints and the utility function (see Formula (4)).

\begin{tabular}{|l|}
\hline $\begin{array}{c}\text { Pefine by } \\
\text { Application? }\end{array}$ \\
\hline$\square$ Macro \\
\hline$\Xi$ Sport \\
\hline$\square$ Portrait \\
\hline$\square$ Landscape \\
\hline$\square$ Architecture \\
\hline \hline Suited for whom? \\
\hline$\square$ beginner \\
\hline$\square$ amateur \\
\hline$\complement$ expert \\
\hline
\end{tabular}

\section{Recommender "Canon DSLR"}
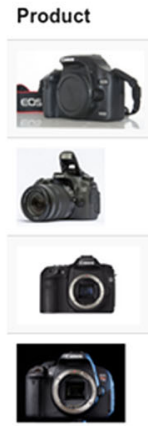

\begin{tabular}{l|l|l|l|l|l}
1 & 2 & 3 & 4 & 5 & ॥
\end{tabular}

Canon EOS 7D

Canon EOS 70D

Canon EOS 50D

Canon EOS 700D
The Canon EOS 7D is a semi-professional cropped sensor digital singlelens reflex camera made by Canon.

The Canon EOS 70D is a digital single-lens reflex camera from Canon.

The Canon EOS 50D combines many of the technologies found in Canon's professional cameras making it the perfect choice f...

The Canon EOS 700D (known as the Kiss X7i in Japan and the Rebel T5i in the Americas) is an 18.0-megapixel digital singl...

Fig. 1 PeOPLEVIEWS user interface: user requirements $\left(r e q_{i} \in R E Q\right)$ are specified on the left hand side, the corresponding recommendations are shown on the right hand side 


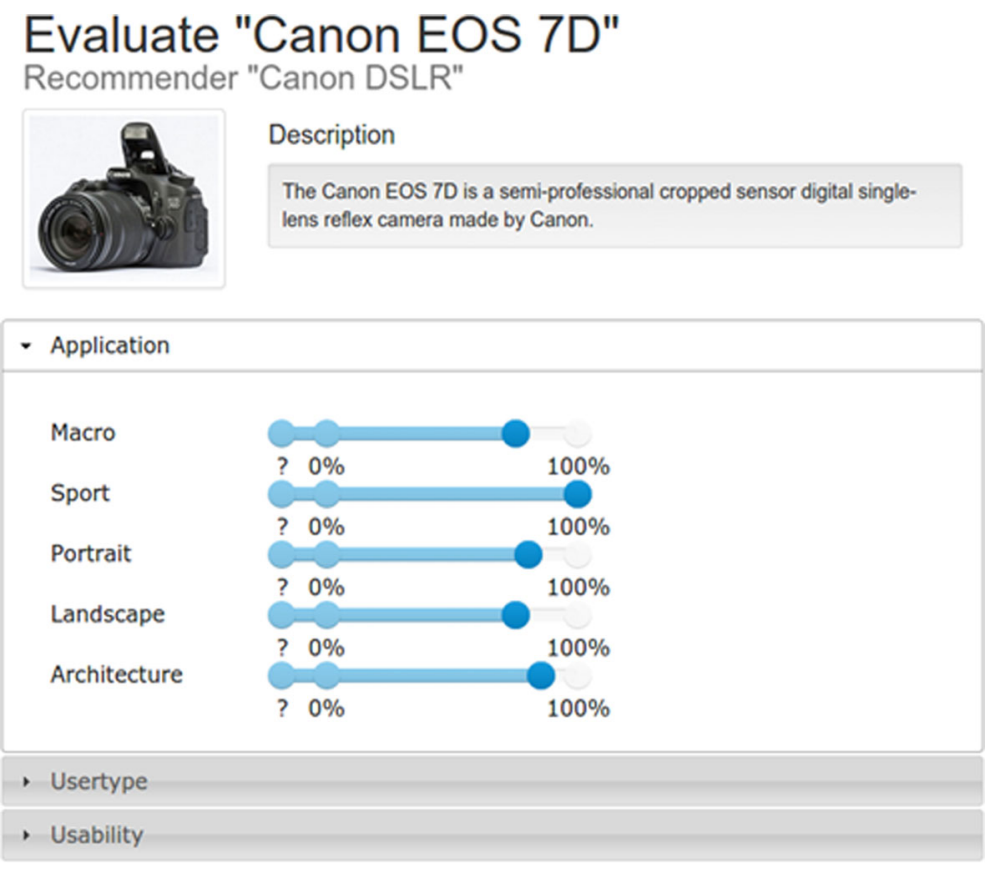

$\checkmark$ Save Cancel

Fig. 2 Evaluation of item Canon EOS $7 D$ with regard to all user attributes $u \in U$

Items in the recommendation list can be selected for product comparison, search criteria entered by the user can be saved, i.e., are taken into account when the user activates the recommender the next time.

Recommenders are created in the PEOPLEVIEWs modeling mode. Figure 2 depicts an interface for the acquisition of a user-specific filter constraint for the item Canon EOS 7D $\left(\Phi_{1}\right)$. The user can evaluate the item with regard to all existing user attributes, however, if the user does not have the knowledge, the corresponding attributes can be omitted. The user input can be directly translated into a corresponding user-specific filter constraint (see, e.g., Table 5). The interface depicted in Fig. 2 can be used when a new item is added or a user wants to evaluate an item.

In the PEOPLEVIEWS modeling mode, there are five different micro-task interfaces (of different complexity) that support the acquisition of knowledge relevant for deriving

Table 7 Micro-task types supported in PEOPLEVIEwS

\begin{tabular}{lll}
\hline id & description & figure \\
\hline 1 & Evaluation one user attribute with regard to one specific value & 3 \\
2 & Selection and evaluation of best-performing item with regard to a specific user attribute value & 4 \\
3 & Evaluation of one single-choice user attribute with regard to all possible values & 5 \\
4 & Evaluation of one multiple-choice user attribute with regard to all possible values & 6 \\
5 & CAPTCHA-style check & 7 \\
\hline
\end{tabular}




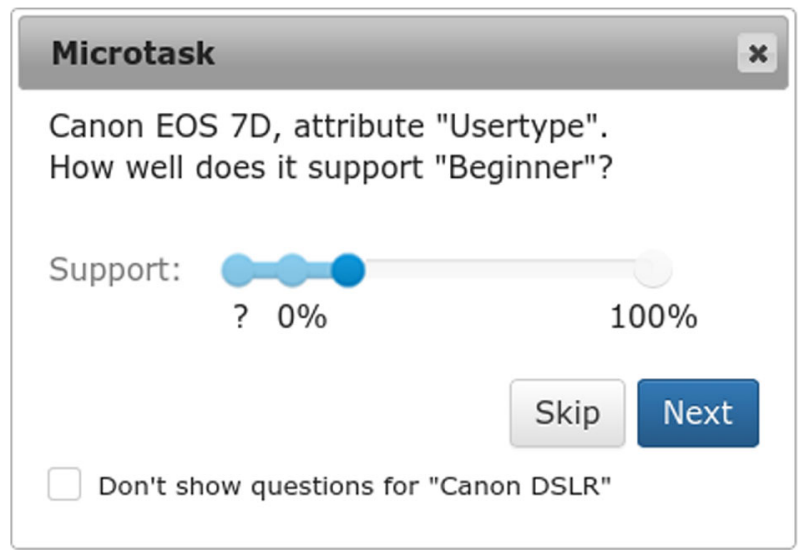

Fig. 3 Evaluation of Canon EOS $7 D$ with regard to a specific user attribute value (usertype=beginner)

user-specific filter constraints (including the corresponding user-specific evaluations). An overview of these micro-tasks is given in Table 7. Figure 3 depicts a simple micro-task interface which supports the acquisition of only one aspect of a user attribute. In this example, the user expresses his/her personal opinion that the item Canon EOS $7 D$ has a very low support regarding the attribute value usertype=beginner.

Similar to Fig. 3, the user interface of Fig. 4 allows the evaluation of item-specific properties. In addition, the interface supports the acquisition of performance relationships between items regarding specific attribute values, for example, information regarding the camera

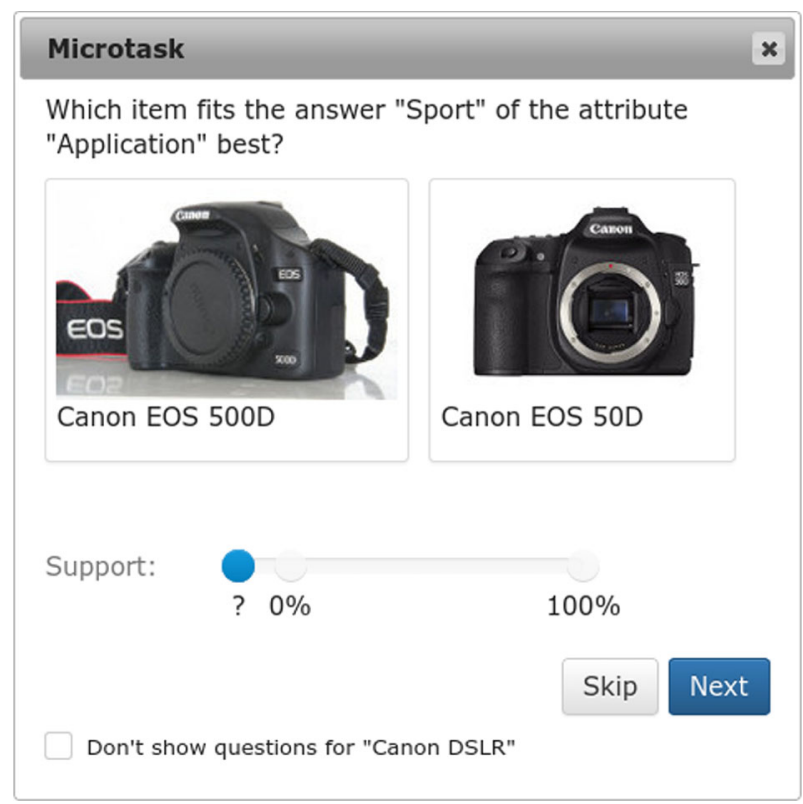

Fig. 4 Evaluation of an item set with regard to a specific user attribute value. Preference relationships regarding item performance are derived as well (e.g., a camera is better with regard to application=sport) 


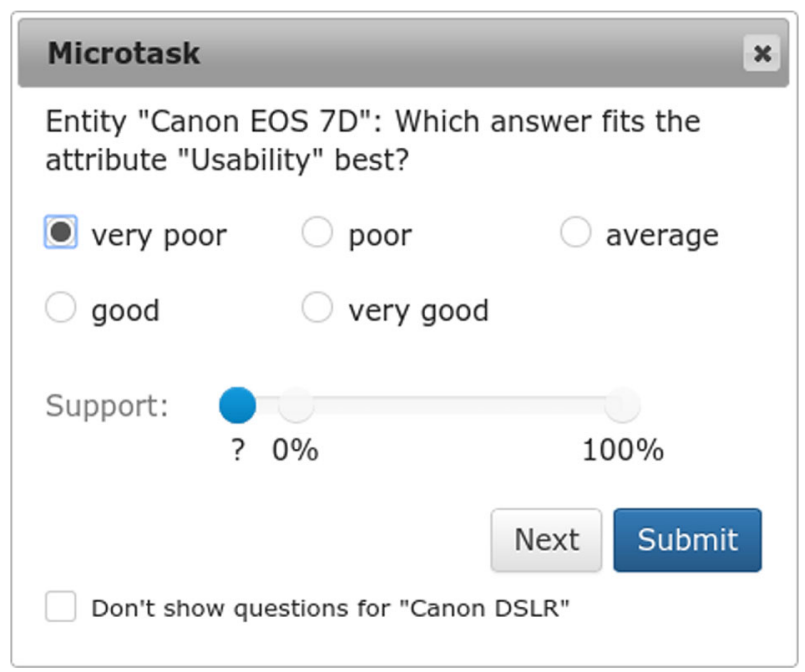

Fig. 5 Evaluation of Canon EOS $7 D$ with regard to a single-choice attribute (usability)

performance in the sports application area. Figure 5 depicts an example of an interface that supports the evaluation of an item with regard to one single choice user attribute (user attribute usability). Figure 6 supports the same functionality for multiple-choice answers (user attribute usertype). Finally, Fig. 7 depicts the user interface of a CAPTCHA-style micro-task which helps to figure out whether the user is a "real" user or a bot.

Gamification-based acquisition of recommendation knowledge One source of recommendation knowledge in PEOPLEVIEWS are user evaluations of item properties performed within the scope of completing micro-tasks. Another source of recommendation knowledge are games where PEOPLEVIEWS users can check their personal item domain

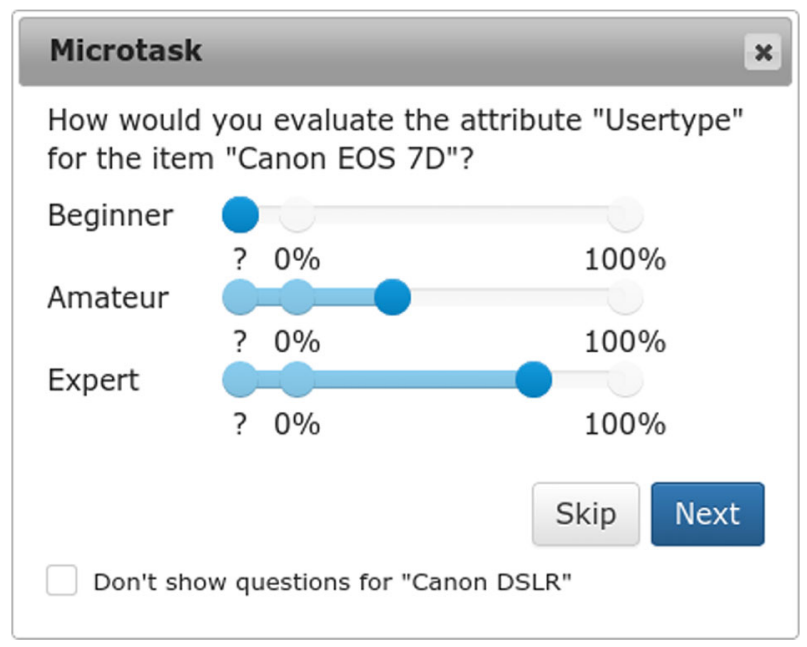

Fig. 6 Evaluation of Canon EOS $7 D$ with regard to a multiple-choice attribute (usertype) 


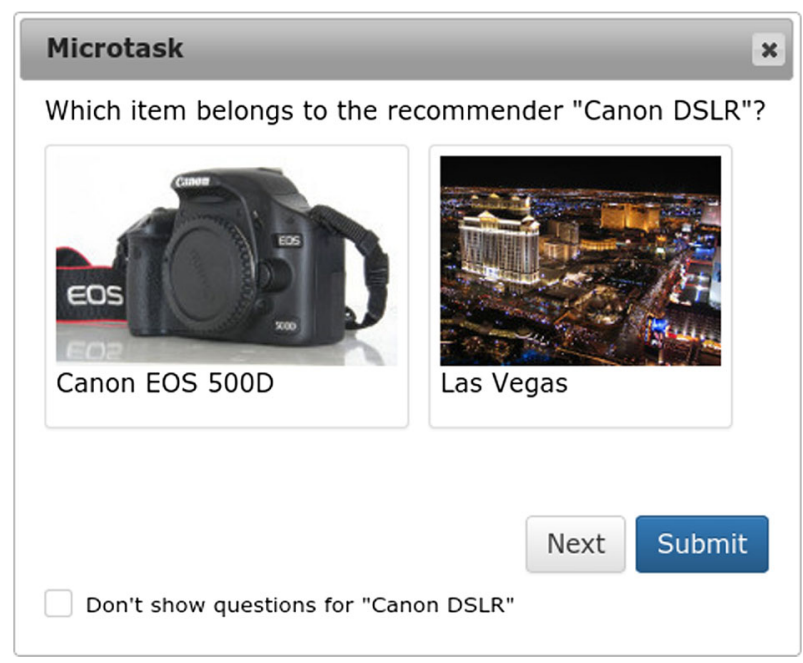

Fig. 7 CAPTCHA-style micro-task

knowledge. Users can either play in groups of two users or in a single-user mode. Two users receive points if they manage to agree on an answer to a question (without seeing the answer of the other user) - the less iterations are needed for achieving the goal, the better. Questions are always related to topics out of the product (recommender) domain (e.g., Canon DSLR cameras) the game is assigned to - users have to pre-select the topic before a game can be started. In the single-user mode, the goal is to answer questions the same way as already done by the majority of other PEOPLEVIEWS users confronted with the same question (posed, e.g., in micro-tasks or prior games). An example of a game in single-user mode is given in Fig. 8. Answers of users (including their item attribute value evaluations) provided within the scope of a gaming session are used in the same way as user answers to micro-tasks.

Inconsistency management When interacting with constraint-based recommenders, users are sometimes confronted with a situation where the recommender is not able to identify a recommendation for a given set of customer requirements. The reason for this is that requirements can induce an inconsistency with the underlying set of constraints. For example, if a user articulates the preferences (requirements) pocket camera and at the same time exchangeable lenses, the recommendation knowledge base will not be able to identify a corresponding solution. Many user interfaces simply inform the user about the situation and indicate that the requirements have to be adapted. PEOPLEVIEWS follows a more sophisticated approach to deal with such a situation: each recommender has a built-in analysis functionality that automatically determines a minimal set of requirements that have to be adapted by the user such that at least one solution can be identified by the recommendation knowledge base. The algorithmic approach to determine such sets of requirements is denoted as FASTDIAG which is a divide-and-conquer based algorithm for the determination of minimal hitting sets - details of the algorithm are discussed in Felfernig et al. (2012).

PeopleViews mobile The PEOPLEVIEWs environment provides an HTML-5 user interface that includes both, the modeling mode (recommenders can be defined on the basis of 


\section{Item Evaluation Game (Single User) Recommender "Canon DSLR" \\ Questions answered

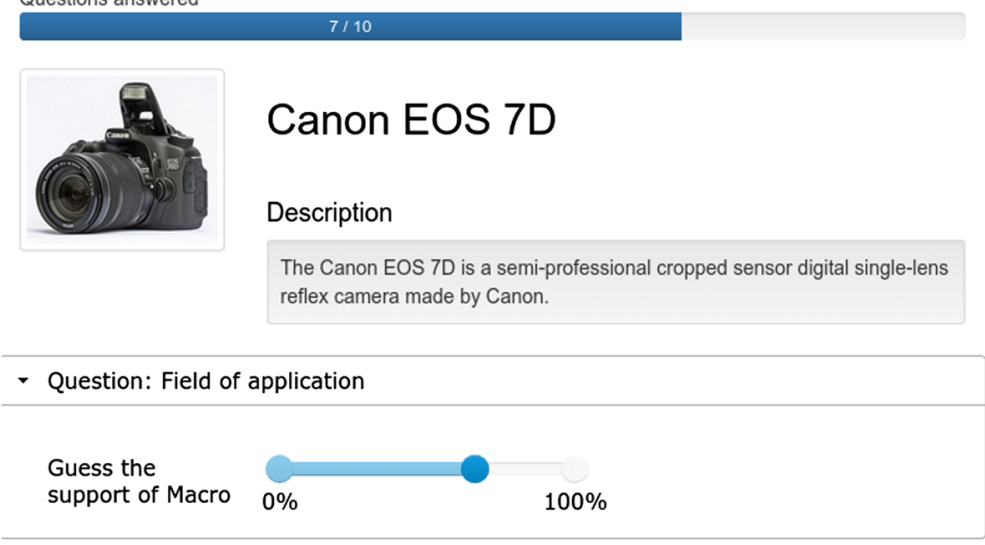

Fig. 8 Item Evaluation Game in single-user mode

micro-tasks) and the recommendation mode. We have also developed an iOS-based mobile version (Promitzer et al. 2016) that supports a subset of the functionalities provided by the HTML-5 version. In the mobile version, users can provide answers to micro-tasks, engage in games, and apply the different recommenders already developed by the PEOPLEVIEWS community. An example of the user interface of the PEOPLEVIEWS mobile version is depicted in Fig. 9.

\section{Evaluation}

In order to evaluate the different recommendation approaches currently integrated in the system, we used a PEOPLEVIEWS dataset created by users with expertise in the domain of digital cameras. Each of the $\mathrm{N}=35$ users had experiences in the Canon DSLR camera domain and performed the following two basic tasks: (1) specification of a set of user requirements related to user attributes selected by the user and selection of a corresponding digital camera that best fulfills these requirements and (2) evaluation of a user-selected set of digital cameras (cameras selected in (1) were not selectable in this context) with regard to the given set of user attributes. The result of task 1 is a set of 45 test cases (100\% coverage of the Canon DSLR cameras is given) that are the basis for evaluating the predictive quality of the integrated recommendation algorithms. The result of task 2 is a set of 285 item evaluations that are the basis for the derivation of a set of recommendation-relevant filter constraints. The study was announced via the online channels of Graz University of Technology. The set of user attributes that could be instantiated by a user in terms of requirements is a superset of those used in our working example (see Table 1): preferred application, photography experiences, usability, cost effectiveness, and allowed loss of value. 
No SIM $~$
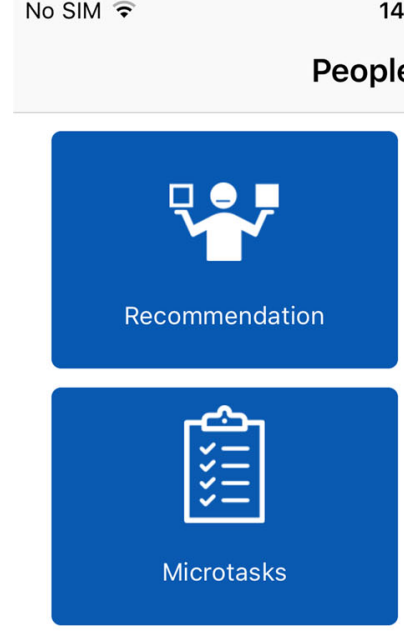

$14: 24$

\section{eopleViews}

\section{PeopleViews rank}

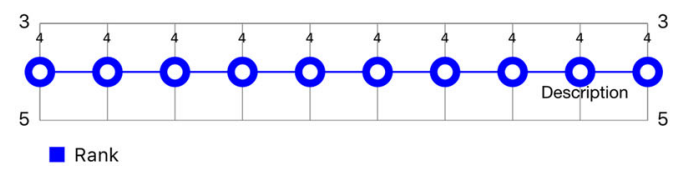

\section{Popular recommenders}

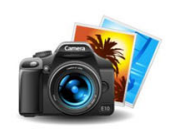

Canon DSLR

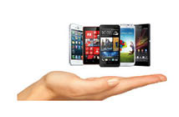

Mobile

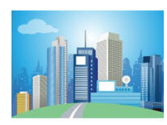

City

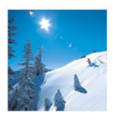

Skiing

Fig. 9 Start-screen of the PEOPLEVIEWS mobile version

The resulting test set (result of task 1) was used for evaluating the predictive quality of the presented recommendation approaches. Using this test set, we compared the baseline methods random (the recommended items were selected randomly), most frequent (the most frequently selected items were recommended), and user similarity based (recommendation of items selected by users with similar preferences) with the ones presented in this paper (basic and extended where the latter is based on beta distributions as discussed in Section 2). Obviously, all except one of the baseline approaches do not take into account the preferences (requirements) defined by the current user.

In order to evaluate our recommendation approaches, we applied $f$-measure and recall (Herlocker et al. 2004) as a metric (see Formulae (8) - (10)) since for digital camera recommendation it is important to find as many relevant items as possible but omit the irrelevant ones (Gunawardana and Shani 2009). In Formulae (8)-(10), \#hits $(N)$ denotes the number of times an item that was selected by a user in the test set was also included in the top-N recommended items. Furthermore, $|D|$ is the size of the test set. The number of hits divided 
by the number of possible hits $(|D|)$ represents the recall. Precision is then defined as the ratio between the number of hits and the number of recommended items which is $N \times|D|$.

$$
\begin{gathered}
f \text {-measure }(N)=2 \times \frac{\operatorname{precision}(N) \times \operatorname{recall}(N)}{\operatorname{precision}(N)+\operatorname{recall}(N)} \\
\operatorname{precision}(N)=\operatorname{recall}(N) \times \frac{1}{N} \\
\operatorname{recall}(N)=\frac{\# \operatorname{hit}(N)}{|D|}
\end{gathered}
$$

Figures 10 and 11 depict a comparison of the predictive quality of the following approaches: random, most frequent, user similarity based, basic, and extended (beta distribution based). Due to the low number of items (\#13 cameras were used in our study), all algorithms achieve nearly $100 \%$ recall for $N=10$. In addition, we also analyze to which extent recommendation performance can be further improved by supporting the learning of the importance weights of user preferences. A comparison of the recommendation approaches with learned weights (see Formula (4)) is depicted in Fig. 12. In this context, weights have been learned on the basis of a genetic algorithm with the goal to improve recall (details on the implementation of this learning approach are provided in Ulz (2016)).

In order to achieve high data quality, it is important to assure that domain experts perform micro-tasks. This can be done by letting users themselves select the items they would like to evaluate or to identify relevant users by analyzing user interaction logs (see also Section 5). When taking into account learned weights (see Fig. 12), a nearly $100 \%$ recall rate can be achieved for the top-3 items which is acceptable for a digital camera recommender (at least one among three recommended items has a very high probability of being accepted by the user). Since only N=35 users participated in our study, further improvements with regard to prediction quality can be expected. The number of potential users who can contribute to micro-tasks is in general limited by the item domain, for example, a recommender for iOS mobile phones has more potential contributors compared to mobile phones with a lower market share.

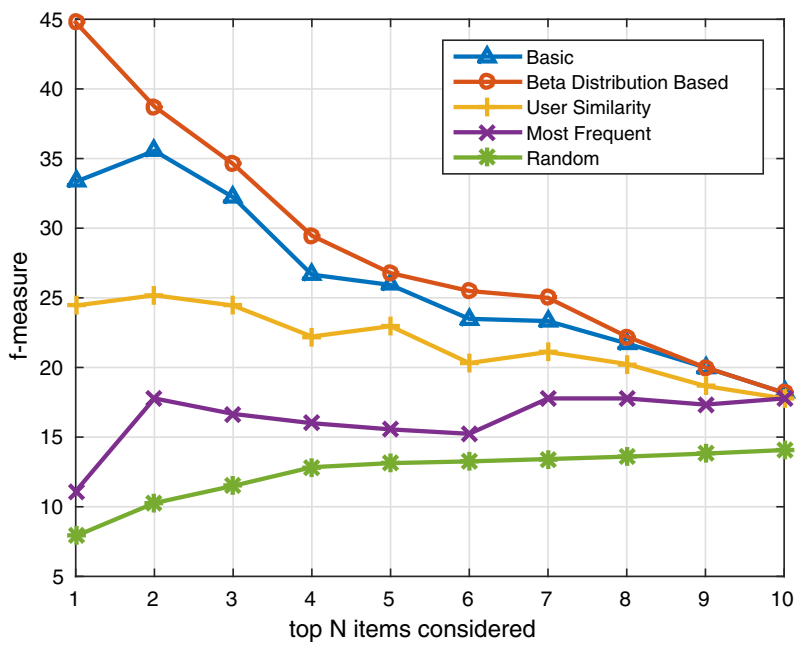

Fig. 10 Comparison of baseline versions (random, most frequent, and user similarity) with PEOPLEVIEWS recommenders (basic and extended) with regard to $f$-measure (see Formula (8)) 


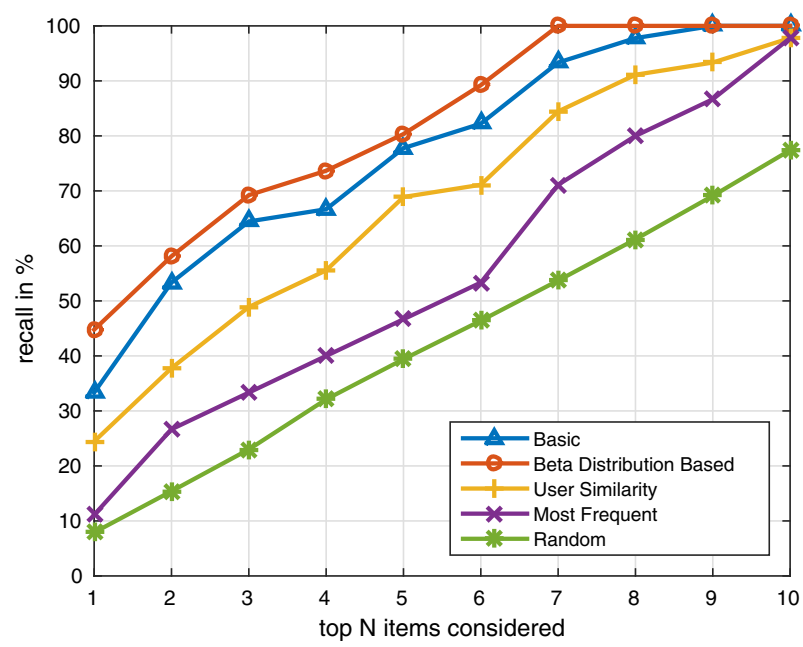

Fig. 11 Comparison of baseline versions (random, most frequent, and user similarity) with PEOPLEVIEws recommenders (basic and extended) with regard to recall (see Formula (10))

\section{Ongoing and future work}

Recommendation approaches The comparison with further recommendation approaches is within the scope of our future work. Using user interaction logs we will compare utility-based approaches with different further approaches to case-based reasoning (CBR) that include, for example, a probability-based approach to determine similarities (Deshpande and Karypis 2004). In this context, we will also compare ensemble-based (hybrid) approaches with regard to their capability of improving prediction quality. Finally, we want to expand the way in which PEOPLEVIEws concepts can be applied in taste

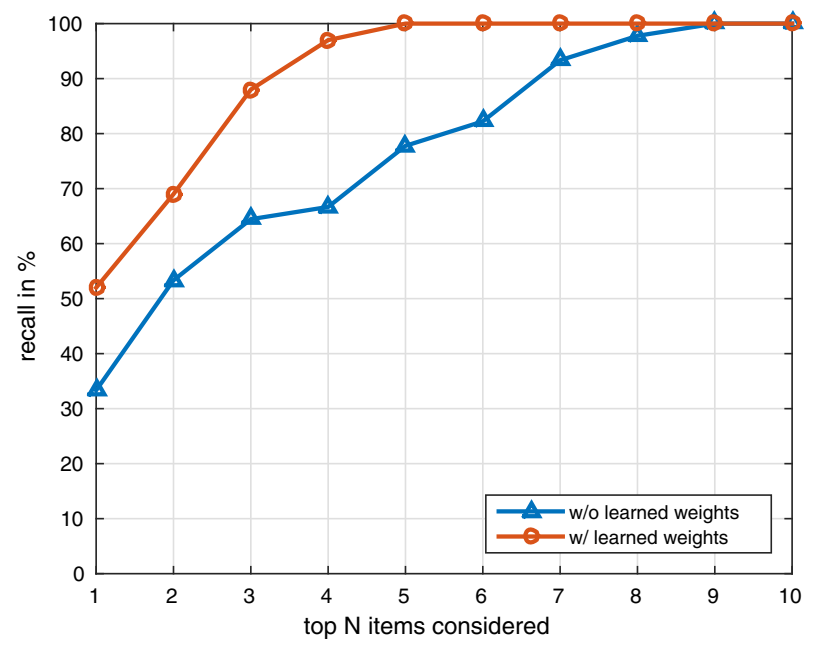

Fig. 12 Comparison of PeopleVIEws recommenders (basic with and without learned weights) with regard to recall (see Formula (10)) 
domains, where there is not a unique, objective truth about items, for example, in the context of collaborative filtering recommendation scenarios (see, e.g., (Larson et al. 2013; Organisciak et al. 2013)).

Usability studies The current version of the PEOPLEVIEWS user interface is the result of a couple of iterations that focused on improving the understandability of the user interface. The different presented types of micro-tasks are the result of this process. However, further studies are needed to continuously improve the usability of the interface, for example, we plan to improve the overall quality of explanations. In this context we also want to identify ways to include the results of micro-tasks into explanation generation. For example, if no solution can be identified for a set of user requirements, the determination of corresponding repair actions can be guided on the basis of user feedback from micro-tasks. In order to achieve this goal, additional micro-task templates have to be developed that extend the existing set.

Micro-task scheduling Recommendation-relevant filter constraints are derived from data collected by micro-tasks. We have already integrated a basic version of a scheduling approach that assigns micro-tasks to users. This is in the line of user-item reciprocity (Larson et al. 2013; Said et al. 2014): (1) users should be interested in items recommended to them and also have the potential to enrich them and (2) mechanisms are needed for enticing users to provide data. The first aspect can be considered as a more traditional recommendation related task whereas the second one includes motivational factors to engage users. In PEOPLEVIEWS, the second aspect is currently covered by a scoring approach were points can be received, for example, for completing micro-tasks, adding new items to a recommender, or evaluating items. For industrial scenarios (e.g., in the financial services domain) we assume the inclusion of different types of motivation mechanisms ranging from monetary approaches to recognitions. These can be based on scoring information from the PEOPleVIEWs environment. This way, PEOPLEVIEWs remains open which is important since mechanisms can differ significantly.

The micro-task scheduling approach currently integrated in PEOPLEVIEWS is based on the dimensions of importance, ability, and interest. We interpret importance of a micro-task as a measure of time, i.e., the more time passed between the creation and distribution of the micro-task and the current time, the more important the task is.

Furthermore, the ability of a user to complete a micro-task is measured in terms of the similarity between the description of the micro-task and the information about micro-tasks that were already successfully completed by the user in the past (information contained in the user profile). Finally, a user's interest in completing a micro-task is measured in terms of the amount of micro-tasks that have already been completed in the early past. We denote the union of ability and interest as qualification of a user to complete a certain micro-task. In PeopleViews, the creation of a micro-task starts with an initial agenda, for example, if a new item has been inserted by a user, a set of micro-tasks is created to evaluate the attributes of the new item. Depending on the quality of the evaluation data, further microtasks need to be created or not (e.g., if there is a uniform-like distribution in the resulting data, further micro-tasks are triggered). We want to emphasize that micro-task generation and user assignment will be further investigated in our future work where we will also take into account existing work in the context of active learning (Elahi et al. 2016).

Quality assurance CAPTCHA-style micro-tasks (see, e.g., Fig. 7) are used to identify micro-task contributions that should not be forwarded to the recommendation process. The 
higher the share of faulty answers of a certain user, the higher the probability that the user is, for example, not a real user but a bot trying to insert item evaluations into the PEOPLEVIEWS environment. Furthermore, timing models indicate to which extent the time efforts of the current user correspond to those of other users. The higher the deviation of the time efforts of the current user, the lower the impact of the corresponding contribution. A basic means to assure the quality of micro-contributions are constraints representing ground truth. In the context of collecting micro-task feedback for a digital camera recommender, some questions can be formulated that help to validate the qualification of a user with regard to a certain topic. If, for example, the question regarding the sensor type included in a Canon EOS 5D Mark III has been answered incorrectly, further contributions of this user are considered of lower value compared to users who answered such questions correctly. One of our goals for future work is to compare the performance of different user communities with regard to the completion of micro-tasks. For the domain of digital cameras we want to compare the recommendation quality of knowledge bases constructed by micro-worker communities such as microworkers.com with expert communities in the domain of digital cameras. The comparison of such types of communities and their properties is our focus for future work.

\section{Conclusions}

In this paper we provide an overview of the PEOPLEVIEWS environment which supports the Human Computation based development of constraint-based recommenders. PEOPLEVIEWS includes five types of micro-tasks that can be used for collecting recommendation knowledge. We introduce the currently integrated recommendation approaches and analyze their prediction quality. Furthermore, we sketch the currently developed approaches to micro-task scheduling and quality assurance of micro-task data. Major issues for future work are the inclusion of additional recommendation algorithms that help to improve prediction quality, detailed empirical studies related to the quality of micro-task scheduling, i.e., selecting and assigning micro-tasks to users, and empirical studies related to the quality of datasets acquired from different user communities.

Acknowledgments Open access funding provided by Graz University of Technology, Graz, Austria.

Open Access This article is distributed under the terms of the Creative Commons Attribution 4.0 International License (http://creativecommons.org/licenses/by/4.0/), which permits unrestricted use, distribution, and reproduction in any medium, provided you give appropriate credit to the original author(s) and the source, provide a link to the Creative Commons license, and indicate if changes were made.

\section{References}

Burke, R. (2000). Knowledge-based Recommender Systems. Encyclopedia of Library and Information Systems, 69(32), 180-200.

Burke, R., \& Hammond, K. (1997). The FindMe Approach to Assisted Browsing. IEEE Expert, 32-40.

Burke, R., \& Ramezani, M. (2010). Matching Recommendation Technologies and Domains. In Recommender Systems Handbook (pp. 367-386): Springer.

Colson, E. (2013). Using human and machine processing in recommendation systems. In human computation and crowdsourcing: Works in progress and demonstration abstracts, Technical Report CR-13-01 (pp. 1617). 
Cosley, D., Frankowski, D., Terveen, L., \& Suggestbot, J.Riedl. (2007). Using intelligent task routing to help people find work in wikipedia. In IUI'07 (pp. 32-41).

Deshpande, M., \& Karypis, G. (2004). Item-Based Top-N Recommendation algorithms. ACM Transactions on Information Systems, 22(1), 143-177.

Elahi, M., Ricci, F., \& Rubens, N. (2016). A survey of active learning in collaborative filtering recommender systems. Computer Science Review, 20, 29-50.

Felfernig, A., \& Burke, R. (2008). Constraint-based Recommender Systems: Technologies and Research Issues. In IEEE ICEC'08, 17-26, Innsbruck, Austria.

Felfernig, A., Friedrich, G., Jannach, D., \& Zanker, M. (2006). An Environment for the Development of Knowledge-based Recommender Applications. International Journal of Electronic Commerce (IJEC), 11(2), 11-34.

Felfernig, A., Haas, S., Schwarz, M., Ulz, T., Stettinger, M., \& Reiterer, S. (2015). Micro-Task Scheduling for Constraint-based Recommendation. In Technical Report, Graz University of Technology, pages 1-6, Graz, Austria.

Felfernig, A., Schubert, M., Friedrich, G., Mandl, M., Mairitsch, M., \& Teppan, E. (2009). Plausible Repairs for Inconsistent Requirements. In 21st International Joint Conference on Artificial Intelligence (IJCAI'09), pages 791-796, Pasadena, CA.

Felfernig, A., Schubert, M., \& Zehentner, C. (2012). An efficient diagnosis algorithm for inconsistent constraint sets. AIEDAM, 25(2), 175-184.

Goldberg, D., Nichols, D., Oki, B., \& Terry, D. (1992). Using collaborative filtering to weave an information tapestry. Communications of the ACM, 35(12), 51-60.

Gunawardana, A., \& Shani, G. (2009). A survey of accuracy evaluation metrics of recommendation tasks. Journal of Machine Learning Research, 10, 2935-2962.

Hacker, S., \& Matchin, L.v.on.Ahn. (2009). Eliciting user preferences with an online game. In CHI'09 (pp. 1207-1216).

Iren, D., \& Bilgen, S. (2014). Cost of quality in crowdsourcing. Human Computation, 1(2), 283-314.

Herlocker, L.T.erveen.J., Konstan, J., \& Riedl, J. (2004). Evaluating collaborative filtering recommender systems. ACM Transactions on Information Systems, 22(1), 5-53.

Jannach, D., \& Bundgaard-Joergensen, U. (2007). SAT: A Web-Based interactive advisor for Investor-Ready business plans. In Intl. Conference on e-Business (ICE-B 2007) (pp. 99-106).

Jannach, D., Zanker, M., Felfernig, A., \& Friedrich, G. (2010). Recommender Systems - An Introduction. Cambridge: Cambridge University Press.

Jung, J. (2014). Quality Assurance in Crowdsourcing via Matrix Factorization based Task Routing. In WWW'14, pages 3-7, Seoul, Korea.

Konstan, J., Miller, B., Maltz, D., Herlocker, J., Gordon, L., \& Riedl, J. (1997). GroupLens: Applying collaborative filtering to usenet news. Comm. of the ACM, 40(3), 77-87.

Koren, Y., Bell, R., \& Volinsky, C. (2009). Matrix factorization techniques for recommender systems. IEEE Computer, 42(8), 30-37.

Larson, M., Said, A., Shi, Y., Cremonesi, P., Tikk, D., \& Karatzoglou, A. (2013). Activating the Crowd: Exploiting User-Item Reciprocity for Recommendation. In CrowdRec'13, pages 1-2, Hong Kong, China.

Leitner, G., Fercher, A., Felfernig, A., \& Hitz, M. (2012). Reducing the Entry Threshold of AAL Systems Preliminary Results from Casa Vecchia. In 13th Intl. Conference on Computers Helping People with Special Needs (pp. 709-715).

McCarthy, K., Reilly, J., Smyth, B., \& McGinty, L. (2005). Generating diverse compound critiques. AI Review, 24(3-4), 339-357.

McSherry, D. (2003). Similarity and compromise. In 5th International Conference on Case-Based Reasoning (pp. 291-305).

Mittal, S., \& Frayman, F. (1989). Towards a Generic Model of Configuration Tasks. In 11th International Joint Conference on Artificial Intelligence (IJCAI'89), pages 1395-1401, Detroit, MI, USA.

Musto, C., Semeraro, G., Lops, P., DeGemmis, M., \& Lekkas, G. (2014). Financial Product Recommendation through Case-based Reasoning and Diversification Techniques. In ACM RecSys, pages 1-2, Foster City, CA, USA.

Nasery, M., Elahi, M., \& Cremonesi, P. (2014). Polimovie: a Feature-based Dataset for Recommender Systems. In CrowdRec'14 (pp. 1-6).

Organisciak, P., Teevan, J., Dumais, S., Miller, R., \& Kalai, A. (2013). Personalized Human Computation. In HCOMP'13, pages 56-57, Palm Springs, CA, USA.

Pazzani, M., \& Billsus, D. (1997). Learning and revising user profiles: The identification of interesting web sites. Machine Learning, 27, 313-331. 
Peischl, B., Zanker, M., Nica, M., \& Schmid, W. (2010). Constraint-based Recommendation for Software Project Effort Estimation. Journal of Emerging Technologies in Web Intelligence, 2(4), 282-290.

Promitzer, A., Felfernig, A., Schwarz, M., Ulz, T., Shehadeh, A., \& Haas, S. (2016). A Short Overview of the PeopleViews Mobile User Interface. In TU Graz Technical Report (pp. 1-4).

Roy, L., \& Mooney, R. (2004). Content-based Book Recommending Using Learning for Text Categorization. User Modeling and User-Adapted Interaction, 14(1), 37-85.

Reiterer, S. (2015). An Integrated Knowledge Engineering Environment for Constraint-based Recommender Systems. In FinRec'15, pages 11-18, Graz, Austria.

Ricci, F., Rokach, L., Shapira, B., \& Kantor, P. (2011). Recommender systems handbook springer.

Said, A., Larson, M., Tikk, D., Cremonesi, P., Karatzoglou, A., Hopfgartner, F., Turrin, R., \& Geurt, J. (2014). User-Item Reciprocity in Recommender Systems: Incentivizing the Crowd. In UMAP Project Synergy Workshop (UMAP ProS'14), pages 23-26, Aalborg, Denmark.

Salem, Y., Hong, J., \& Liu, W. (2014). History-guided Conversational Recommendation. In 23rd International Conference on World Wide Web (WWW'14), pages 999-1004. ACM.

Shah, N., \& Zhou, D. (2015). On the impossibility of convex inference in human computation. In AAAI'15 (pp. 1291-1297).

Torrens, M., Hertzog, P., Samson, L., \& Faltings, B. (2003). Reality: A Scalable Intelligent Travel Planner. In ACM Symp. on Applied Computing, pages 623-630, Melbouurne, FL, USA.

Ulz, T. (2016). Human Computation based Recommendation Technologies. In Master Thesis, Graz University of Technology, pages 1-103, Graz, Austria.

von Ahn, L. (2005). Human Computation. In Technical Report CM-CS-05-193.

Walsh, G., \& Golbeck, J. (2009). Curator: A Game with a Purpose for Collection Recommendation. In CHI 2009, pages 2079-2082, Boston, Massachusetts, USA.

Winterfeldt, D., \& Edwards, W. (1986). Decision analysis and behavioral research, Cambridge University Press, Cambridge.

Zanardi, V., \& Cara, L. (2008). Social Ranking: Uncovering Relevant Content Using Tag-based Recommender Systems. In Recommender Systems, (Vol. 2008 pp. 51-58). 